\title{
Physics cases of rare B meson decays at Belle II
}

\author{
Katsuro Nakamura* \\ Author: High Energy Accelerator Research Organization (KEK) \\ E-mail: katsuro.nakamura@kek.jp \\ on behalf of the Belle II Collaboration
}

Flavor changing neutral current decays of $B$ mesons are powerful probes for the new physics search. At the Belle II experiment, these decay modes will be precisely studied with a high integrated luminosity of $50 \mathrm{ab}^{-1}$ which is 50 times higher than the luminosity of Belle. Radiative and semi-leptonic penguin $B$ decays are important channels especially for the new physics search because they are strongly suppressed in the standard model. In this article, projections of uncertainties on the radiative and semi-leptonic penguin decay measurements are reported.

XIV International Conference on Heavy Quarks and Leptons (HQL2018)

May 27- June 1, 2018

Yamagata Terrsa, Yamagata,Japan

${ }^{*}$ Speaker. 


\section{Introduction}

Belle II is an electron-positron collision experiment upgraded from the predecessor experiment Belle. At Belle II, we search for physics beyond the standard model (BSM) with an integrated luminosity of $50 \mathrm{ab}^{-1}$ which is provided by the SuperKEKB accelerator. This integrated luminosity is 50 times higher than the luminosity of Belle. For the BSM search, flavor changing neutral current (FCNC) processes in $b \rightarrow s$ and $b \rightarrow d$ decays are powerful probes, because they are well suppressed in the standard model while they can be enhanced in the BSM. In the FCNC $B$ decay channels, radiative penguin decay and semi-leptonic penguin decay are important channels. In this article, expected precision of several interesting measurements in those decays are summarized.

\section{Radiative penguin $B$ decays}

\subsection{Branching fraction of $B \rightarrow X_{s} \gamma$}

The inclusive radiative penguin $B$ decay, $B \rightarrow X_{s} \gamma$, provides constraints on many possible BSM scenarios. One good example is the current strong constraint on the two Higgs doublet model (2HDM) from the measured branching fraction of $B \rightarrow X_{s} \gamma$. The current world-average of the $b \rightarrow s \gamma$ branching fraction from the $B$-factory measurements is $\mathscr{B}_{s \gamma}^{\exp }\left(E_{\gamma}>1.6 \mathrm{GeV}\right)=$ $(3.49 \pm 0.19) \times 10^{-4}$ [四]. The result excludes charged-Higgs mass less than $580 \mathrm{GeV}$ in the $2 \mathrm{HDM}$ type-II model with a $95 \%$ confidence level [ᄆ]].

The newest Belle result of the $B \rightarrow X_{s} \gamma$ branching ratio with fully inclusive method gives $\mathscr{B}_{s \gamma}^{\text {Belle }}\left(E_{\gamma}>1.6 \mathrm{GeV}\right)=(3.12 \pm 0.10$ (stat) \pm 0.19 (syst) \pm 0.08 (model) $\pm 0.04($ extrap $)) \times 10^{-4}$ [ [B] . This uncertainty is dominated almost by the systematic uncertainty, which is attributed to uncertainties on the $B$ meson pair counts, detector response, background subtraction, and fragmentation model. Therefore, the mission at Belle II is to reduce the systematic uncertainties with the improved statistics. One advantage of the $\mathscr{B}_{s \gamma}$ measurement at Belle II is that the branching fraction with $E_{\gamma}>1.6 \mathrm{GeV}$ can be measured without extrapolation for $E_{\gamma}$. Expected total uncertainty on the $\mathscr{B}_{s \gamma}$ measurement at Belle II is $3.9 \%$ with the $50 \mathrm{ab}^{-1}$ integrated luminosity. This uncertainty is comparable to a theoretical uncertainty due to non-perturbative effect.

\subsection{Direct $C P$ asymmetry of $B \rightarrow X_{(s, d)} \gamma$}

The baryon asymmetry in the universe indicates existence of new physics with $C P$ violation. Hence, $C P$ asymmetry measurement in rare $B$ decays is a powerful probe to search for the new physics.

Direct $C P$ asymmetry (time-integral) of the inclusive radiative decay is defined as

$$
A_{C P}(s \gamma)=\frac{\Gamma\left(\bar{B} \rightarrow X_{s} \gamma\right)-\Gamma\left(B \rightarrow X_{\bar{s}} \gamma\right)}{\Gamma\left(\bar{B} \rightarrow X_{s} \gamma\right)+\Gamma\left(B \rightarrow X_{\bar{s}} \gamma\right)} .
$$

The standard model predicts that the direct $C P$ asymmetries for $B \rightarrow X_{s} \gamma$ and $B \rightarrow X_{d} \gamma$ can be non-zero due to a long-distance $c$-loop effect [四, 目], which has large theoretical uncertainties, $A_{C P}^{\mathrm{SM}}(s \gamma)=[-0.6,2.8] \%, A_{C P}^{\mathrm{SM}}(d \gamma)=[-62,14] \%$. On the other hand, due to the CKM unitarity the direct $C P$ asymmetry for the sum of $s$ and $d$ decays $B \rightarrow X_{s+d} \gamma$ is predicted to be small as $\sim \Lambda_{\mathrm{QCD}} / m_{b}$ in the standard model. The asymmetry measurement is sensitive for BSM which can 
create larger asymmetry than this prediction. Moreover, difference of $A_{C P}$ between charged and neutral $B$ mesons

$$
\Delta A_{C P}\left(B \rightarrow X_{s} \gamma\right)=A_{C P}\left(B^{+} \rightarrow X_{s}^{+} \gamma\right)-A_{C P}\left(B^{0} \rightarrow X_{s}^{0} \gamma\right),
$$

also has good sensitivity to BSM, because the difference is proportional to $\operatorname{Im}\left(C_{8 g} / C_{7 \gamma}\right)$, which is zero in the standard model.

Existing $A_{C P}\left(B \rightarrow X_{s+d} \gamma\right)$ measurements for $E_{\gamma}>2.1 \mathrm{GeV}$ done by Belle and BaBar with the sum-of-exclusive method find $(0.2 \pm 5.0 \pm 3.0) \%$ and $(1.7 \pm 1.9 \pm 1.0) \%$, respectively [四]. $\Delta A_{C P}\left(B \rightarrow X_{s} \gamma\right)$ measurement for $E_{\gamma}>2.1 \mathrm{GeV}$ at BaBar finds $(5.0 \pm 3.9 \pm 1.5) \%$ [目].

At Belle II we will measure $A_{C P}\left(B \rightarrow X_{s+d} \gamma\right)$ and $\Delta A_{C P}\left(B \rightarrow X_{s} \gamma\right)$ precisely. The statistical uncertainties and some systematic uncertainties due to the detector asymmetry and an asymmetry created by the background also can be improved with the higher statistics. Hence, we expect uncertainties on $A_{C P}\left(B \rightarrow X_{s+d} \gamma\right)$ and $\Delta A_{C P}\left(B \rightarrow X_{s} \gamma\right)$ measurement at Belle II will be dominated by statistical even after the designed integrated luminosity $50 \mathrm{ab}^{-1}$ is achieved. Expected uncertainties on $A_{C P}\left(B \rightarrow X_{s+d} \gamma\right)$ is $\pm 0.6 \%$ with $50 \mathrm{ab}^{-1}$. This corresponds to $3.7 \sigma$ significance of the $C P$ violation discovery, if the current central value of $A_{C P}\left(B \rightarrow X_{s+d} \gamma\right)$ does not change. Expected uncertainty on $\Delta A_{C P}\left(B \rightarrow X_{s} \gamma\right)$ is $\pm 0.4 \%$ with $50 \mathrm{ab}^{-1}$.

\subsection{Time-dependent $C P$ asymmetry of $b \rightarrow s \gamma$}

As well as the direct $C P$ asymmetry, the time-dependent $C P$ asymmetry in exclusive $b \rightarrow s \gamma$ $C P$-eigenstate is also an excellent probe for the BSM search. The time-dependent $C P$ asymmetry is parameterized with $S_{f \gamma}$ and $C_{f \gamma}$ as in the following definition,

$$
\frac{\Gamma\left[\bar{B}^{0} \rightarrow f \gamma\right](\Delta t)-\Gamma\left[B^{0} \rightarrow f \gamma\right](\Delta t)}{\Gamma\left[\bar{B}^{0} \rightarrow f \gamma\right](\Delta t)+\Gamma\left[B^{0} \rightarrow f \gamma\right](\Delta t)}=S_{f \gamma} \sin (\Delta t)-C_{f \gamma} \cos (\Delta t)
$$

In the standard model, the time-dependent $C P$ violation of the radiative decay is caused by a combination of the $B-\bar{B}$ mixing and the helicity flip in the left-handed current for the $b \rightarrow s \gamma$ decay. Thus, the asymmetry in the standard model is small. If the new physics contains right-handed current, the BSM can be detected as a $C P$ asymmetry. Taking into account the long-distance $c$-loop effect [ $[\mathbf{]}]$, the standard model predictions are found to be

$$
S^{\mathrm{SM}}\left(K^{\star 0} \gamma\right)=(-2.3 \pm 1.6) \%, \quad S^{\mathrm{SM}}\left(\rho^{0} \gamma\right)=(0.2 \pm 1.6) \% .
$$

$B \rightarrow K^{\star 0}\left(\rightarrow K_{\mathrm{S}} \pi^{0}\right) \gamma$ is one of golden modes for the time-dependent $C P$ violation measurement. At Belle II, significant improvements from Belle are foreseen in the $K_{\mathrm{S}} \pi^{0} \gamma$ analysis due to the following upgrades. Firstly, a larger outer radius of the vertex detector (from $6 \mathrm{~cm}$ at Belle to $11.5 \mathrm{~cm}$ at Belle II) is employed. Due to this change, $30 \%$ more $K_{\mathrm{S}}$ with vertex information becomes available. Secondly, the effective tagging efficiency is improved by $13 \%$ or more. Considering these improvements, expected uncertainties for the time-dependent $C P$ measurement at Belle II are $3.0 \%$ for $S_{K_{\mathrm{S}} \pi^{0} \gamma}$ and $6.4 \%$ for $S_{\rho^{0} \pi^{0} \gamma}$. These uncertainties are dominated by statistical uncertainty.

\subsection{Summary of radiative rare decay measurement}

As a summary, the expected uncertainties of the radiative rare decay measurements are listed

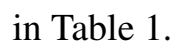




\begin{tabular}{lcc}
\hline Observables & Belle II 5 ab & Belle II 50 ab $\mathrm{ab}^{-1}$ \\
\hline $\mathscr{B}_{s \gamma \text { inc }}^{\text {lep-tag }}$ & $4.8 \%$ & $3.9 \%$ \\
$\mathscr{B}_{s \gamma \text { inc }}^{\text {had }}$ & $7.0 \%$ & $4.2 \%$ \\
$\mathscr{B}_{s \gamma}^{\text {sum-of-ex }}$ & $7.3 \%$ & $5.7 \%$ \\
$A_{C P}\left(B \rightarrow X_{s+d} \gamma\right)_{\text {inc }}^{\text {lep-tag }}$ & $1.5 \%$ & $0.48 \%$ \\
$A_{C P}\left(B \rightarrow X_{s+d} \gamma\right)_{\text {inc }}^{\text {had }}$ & $2.2 \%$ & $0.70 \%$ \\
$\Delta A_{C P}\left(B \rightarrow X_{s} \gamma\right)_{\text {sum-of-ex }}$ & $1.2 \%$ & $0.37 \%$ \\
$S\left(B \rightarrow K^{\star 0} \gamma\right)$ & $9.0 \%$ & $3.0 \%$ \\
$S\left(B \rightarrow \rho^{0} \gamma\right)$ & $19 \%$ & $6.4 \%$ \\
\hline
\end{tabular}

Table 1: Expected uncertainties of radiative rare decay measurements at Belle II with the integrated luminosity of $5 \mathrm{ab}^{-1}$ and $50 \mathrm{ab}^{-1}$.

\section{Semi-leptonic penguin $B$ decays}

\subsection{Branching fraction and angular analysis of $b \rightarrow s l^{+} l^{-}$}

For the angular analysis of $B \rightarrow K^{\star} l^{+} l^{-}$, parameters $P_{i=4,5,6,8}^{\prime}$ are useful quantities to be measured since the theoritical uncertainties from form factors are canceled out for leading order. These parameters are sensitive to Wilson coefficients $C_{7}, C_{9}$, and $C_{10}$. Especially for the $P_{5}^{\prime}$ measurement, $\mathrm{LHCb}$ reported $3.7 \sigma$ tension from the standard model prediction with $1 \mathrm{fb}^{-1}$ data [ [ $]$ ].

Expected uncertainties of the $P_{5}^{\prime}$ measurement at Belle II $50 \mathrm{ab}^{-1}$ are 0.054 for $1<q^{2}<$ $2.5 \mathrm{GeV}^{2}, 0.049$ for $2.5<q^{2}<4 \mathrm{GeV}^{2}$, and 0.040 for $q^{2}>14.2 \mathrm{GeV}^{2}$. The precision is about $20 \%$ lower than expected precision of LHCb $P_{5}^{\prime}$ measurement with $50 \mathrm{fb}^{-1}$ integrated luminosity.

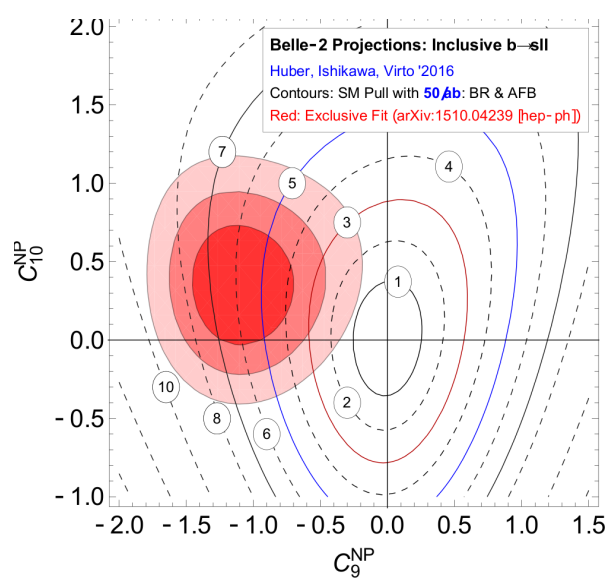

Figure 1: Projection of exclusion contours in the $C_{9}^{N P}-C_{10}^{N P}$ plane resulting from the Belle II $B \rightarrow X_{s} l^{+} l^{-}$ measurements. The central value is assumed to the standard model prediction $\left(C_{9}^{N P}, C_{10}^{N P}\right)=(0,0)$. The red regions are the constraints from the global fit with the current measurement data [Q]].

$B \rightarrow X_{s} l^{+} l^{-}$is a theoretically clean channel compared to exclusive decays, as hadronic uncertainties are under better theoretical control. The branching fraction and angular distribution of $B \rightarrow X_{s} l^{+} l^{-}$are sensitive to $C_{9}$ and $C_{10}[\mathbb{[ 1 0}]$. At Belle II, the branching fraction $d \mathrm{BF} / d q^{2}$ and 
forward-backward asymmetry $A_{\mathrm{FB}}$ in $B \rightarrow X_{s} l^{+} l^{-}$will be measured. Figure $\mathbb{U}$ shows expected constraints on the $C_{9}^{N P}-C_{10}^{N P}$ plane from the branching fraction and forward-backward asymmetry measurements at Belle II.

\subsection{Lepton universality test}

Lepton flavor universality of $b \rightarrow s l^{+} l^{-}$decays will be studied via measurements of the ratio between the branching fractions of the muon and electron decay channels,

$$
R_{X_{s}}=\frac{B R\left(B \rightarrow X_{s} \mu \mu\right)}{B R\left(B \rightarrow X_{s} e e\right)} .
$$

These ratios are sensitive to Wilson coefficients $C_{9}$ and $C_{10}$. The standard model predicts $R \sim$ 1 for $q^{2} \gg m_{\mu}^{2}$. LHCb reported $2.6 \sigma$ deviation from the standard model prediction on the $R_{K}$ measurement [ए]].

There are several advantages at Belle II in the $R_{K^{(\star)}}$ measurement. First, Belle II has an excellent momentum resolution for both electrons and muons. At $\mathrm{LHCb} R_{K^{(\star)}}$ is measured only in low- $q^{2}$, while at Belle II both the low- $q^{2}$ and high- $q^{2}$ regions are accessible. The dominant systematic uncertainty is the uncertainty on lepton identification which is expected to be $0.4 \%$ (relative error). Due to the small systematic uncertainties, the measurement uncertainty at Belle II will be statistically dominated. Moreover, as well as $R_{K^{(\star)}}$, Belle II can measure also the ratio for the inclusive $B \rightarrow X_{s} l^{+} l^{-}$decay, $R_{X_{s}}$.

With $20 \mathrm{ab}^{-1}$ Belle II data, about $5 \%$ uncertainty will be achieved. With this precision the current $R_{K}$ anomaly can be confirmed with a $5 \sigma$ significance, if the central value does not change. With $50 \mathrm{ab}^{-1}$, about $3 \%$ uncertainty can be achieved. Even at the designed integrated luminosity, the uncertainties are statistically limited.

Lepton flavor asymmetry in angular distribution parameters $Q_{i}=P_{i}^{\mu}-P_{i}^{e}$ are also interesting measurements at Belle II. The first measurement of $Q_{4,5}$ was reported by Belle [एँ2]. Belle II will improve the precision. Expected uncertainty on the $Q_{5}$ measurement in the $4<q^{2}<6 \mathrm{GeV}^{2}$ region is 0.040 with $50 \mathrm{ab}^{-1}$ of data.

\section{3 $B \rightarrow K^{(\star)} v \bar{v}$ decay}

$B \rightarrow K^{(\star)} V \bar{v}$ decays are theoretically clean channels and therefore good probes for the BSM search. Main theoretical uncertainties on $B \rightarrow K^{(\star)} v \bar{v}$ are due to the $B \rightarrow K^{(\star)}$ form factor and knowledge of CKM elements. The branching fractions in the standard model are predicted to be $\mathrm{BF}\left(B \rightarrow K^{\star} v \bar{v}\right)_{\mathrm{SM}}=(9.6 \pm 0.9) \times 10^{-6}$ and $\mathrm{BF}\left(B^{+} \rightarrow K^{+} v \bar{v}\right)_{\mathrm{SM}}=(4.5 \pm 0.5) \times 10^{-6}$ which are one order larger than $b \rightarrow$ sll due to the Weinberg angle and the sum of the 3 neutrino flavors in the final state. The branching fraction measurement provides constraints on $C_{L}$ and $C_{R} \cdot \operatorname{BF}(B \rightarrow$ $K^{(\star)} v \bar{v}$ ) can be measured at Belle II $50 \mathrm{ab}^{-1}$ with about $10 \%$ uncertainty. Moreover, a longitudinal polarization $F_{L}$ can also be measured at Belle II with about 0.08 uncertainty.

\subsection{Summary of semi-leptonic penguin $B$ decays}

The expected uncertainties of the semileptonic penguin decay measurements are listed in Table ఐ. 


\begin{tabular}{lcc}
\hline Observables & Belle II 5 ab & Belle II 50 ab \\
\hline$P_{5}^{\prime}\left(1<q^{2}<2.5 \mathrm{GeV}^{2}\right)$ & 0.17 & 0.054 \\
$P_{5}^{\prime}\left(2.5<q^{2}<4 \mathrm{GeV}^{2}\right)$ & 0.15 & 0.049 \\
$P_{5}^{\prime}\left(4<q^{2}<6 \mathrm{GeV}^{2}\right)$ & 0.12 & 0.040 \\
$P_{5}^{\prime}\left(q>14.2^{2} \mathrm{GeV}^{2}\right)$ & 0.088 & 0.027 \\
$R_{K}\left(1<q^{2}<6 \mathrm{GeV}^{2}\right)$ & $11 \%$ & $3.6 \%$ \\
$R_{K}\left(q^{2}>14.2 \mathrm{GeV}^{2}\right)$ & $12 \%$ & $3.6 \%$ \\
$R_{K^{\star}}\left(1<q^{2}<6 \mathrm{GeV}^{2}\right)$ & $10 \%$ & $3.2 \%$ \\
$R_{K^{\star}}\left(q^{2}>14.2 \mathrm{GeV}^{2}\right)$ & $9.2 \%$ & $2.8 \%$ \\
$R_{X_{s}}\left(1<q^{2}<6 \mathrm{GeV}^{2}\right)$ & $12 \%$ & $4.0 \%$ \\
$R_{X_{s}}\left(q^{2}>14.2 \mathrm{GeV}^{2}\right)$ & $11 \%$ & $3.4 \%$ \\
$Q_{5}\left(1<q^{2}<2.5 \mathrm{GeV}^{2}\right)$ & 0.17 & 0.054 \\
$Q_{5}\left(2.5<q^{2}<4 \mathrm{GeV}^{2}\right)$ & 0.15 & 0.049 \\
$Q_{5}\left(4<q^{2}<6 \mathrm{GeV}^{2}\right)$ & 0.12 & 0.040 \\
$Q_{5}\left(q>14.2^{2} \mathrm{GeV}^{2}\right)$ & 0.099 & 0.032 \\
$B\left(B^{+} \rightarrow K^{+} v \bar{v}\right)$ & $30 \%$ & $11 \%$ \\
$B\left(B^{0} \rightarrow K^{\star 0} v \bar{v}\right)$ & $26 \%$ & $9.6 \%$ \\
$B\left(B^{+} \rightarrow K^{\star+} v \bar{v}\right)$ & $25 \%$ & $9.3 \%$ \\
$F_{L}\left(B^{0} \rightarrow K^{\star 0} v \bar{v}\right)$ & - & 0.079 \\
$F_{L}\left(B^{+} \rightarrow K^{\star+} v \bar{v}\right)$ & - & 0.077 \\
\hline
\end{tabular}

Table 2: Expected uncertainties of semileptonic penguin decay measurements at Belle II with the integrated luminosity of $5 \mathrm{ab}^{-1}$ and $50 \mathrm{ab}^{-1}$.

\section{References}

[1] M. Tanabashi et al. (Particle Data Group), Phys. Rev. D 98, 030001 (2018)

[2] A. Arbey, F. Mahmoudi, O. Stal, T. Stefaniak, Eur. Phys. J. C78 (2018) no.3, 182 [arXiv:1706.07414]

[3] Belle Collaboration, BELLE-CONF-1606. [arXiv:1608.02344]

[4] T. Hurth, E. Lunghi, W. Porod, Nucl. Phys. B704 (2005) 56-74 [arXiv:hep-ph/0312260]

[5] M. Benzke, S.J. Lee, M. Neubert, G. Paz, Phys. Rev. Lett. 106 (2011) 141801 [arXiv:1012.3167]

[6] J. P. Lees et al., BaBar, Phys. Rev. D90 (2014) 092001 [arXiv:1406.0534]

[7] P. Ball, R. Zwicky, Phys. Lett. B642 (2006) 478-486 [arXiv:hep-ph/0609037], P. Ball, G.W. Jones, R. Zwicky, Phys. Rev. D75 (2007) 054004 [arXiv:hep-ph/0612081]

[8] Roel Aaij et al. (LHCb collaboration), JHEP, 02, 104 (2016) [arXiv:1512.04442]

[9] Sebastien Descotes-Genon, Lars Hofer, Joaquim Matias, and Javier Virto, JHEP, 06, 092 (2016) [arXiv:1510.04239]

[10] Keith S. M. Lee, Zoltan Ligeti, Iain W. Stewart, and Frank J. Tackmann, Phys. Rev. D75 (2007) 034016, [arXiv:hep-ph/0612156]

[11] R. Aaij et al. (LHCb collaboration), Phys. Rev. Lett. 113 (2014) 151601

[12] S. Wehle et al. (Belle collaboration), Phys. Rev. Lett. 118 (2017) 111801 [arXiv:1612.05014] 